\title{
Quality Control of Mutton by Using Volatile Compound Fingerprinting Techniques and Chemometric Methods
}

\author{
Ping Zhan, ${ }^{1,2}$ Honglei Tian, ${ }^{1,3}$ Baoguo Sun, ${ }^{3}$ Yuyu Zhang, ${ }^{3}$ and Haitao Chen ${ }^{3}$ \\ ${ }^{1}$ College of Food Engineering and Nutritional Science, Shaanxi Normal University, Xi'an, Shaanxi 710119, China \\ ${ }^{2}$ Food College of Shihezi University, Shihezi, Xinjiang 832000, China \\ ${ }^{3}$ School of Food and Chemical Engineering, Beijing Technology and Business University, Beijing 102488, China
}

Correspondence should be addressed to Honglei Tian; thl0993@sina.com and Baoguo Sun; sunbg@btbu.edu.cn

Received 12 June 2017; Accepted 6 August 2017; Published 24 September 2017

Academic Editor: Yuxia Fan

Copyright (C) 2017 Ping Zhan et al. This is an open access article distributed under the Creative Commons Attribution License, which permits unrestricted use, distribution, and reproduction in any medium, provided the original work is properly cited.

\begin{abstract}
A method for chromatographic fingerprinting of flavor was established for the quality control of mutton. Twenty-five mutton samples that were chosen from twelve batches were investigated by gas chromatography-mass spectroscopy (GC-MS) and gas chromatography-olfactometry (GC-O). Spectral correlative chromatograms combined with GC-O assessment were employed, and 32 common odor-active compounds that characterize mutton flavor fingerprint were obtained. Based on the flavor chromatographic fingerprint data, principal component analysis (PCA) and partial least squares-discriminant analysis (PLS-DA) were designed and employed as chromatographic fingerprint methods. Defined categories were perfectly discriminated after PLS-DA was conducted on the fused matrix, demonstrating a 100\% accurate classification. Fourteen constituents were further screened with PLS-DA to be the main chemical markers, and they were used to develop similar approaches for the determination of mutton quality and traceability. The flavor fingerprint of mutton established using SPME-GC-MS/O coupled with PLS-DA is appropriate for differentiating and identifying samples, and the procedure would be used in quality control.
\end{abstract}

\section{Introduction}

Mutton is very popular in Western countries and is widely consumed because of its unique flavor and its nutritional qualities [1]. Meat flavor is mainly produced by the interactions of water-soluble compounds and lipids [2]. Watersoluble compounds, which are present in lean meat, generate the basic meat flavor. Lipids (meat fat) impart the characteristic flavor of distinct meat species [3]. Studies have shown that if fat was removed from meat, meat species are difficult to distinguish based only on their sensory attributes [4]. As such, mutton is often adulterated by low-priced meats (e.g., duck or pork) combined with mutton fat to maximize profit. A continuous supply of adulterated mutton products is mixed with real mutton, resulting in the gradual deterioration of consumer confidence on mutton industry. Therefore, reliable and accurate methods should be established for the quality control of mutton.

Mutton is a complex system with multiple components [5]. Thus, its authenticity and quality cannot be effectively evaluated by simply measuring one or few compounds or indices. Prior to this study, no report has been published on quality control of mutton. Aroma is one of the most important aspects and composition indicators of meat flavor [6]. The sensory attributes of adulterated mutton samples are similar to those of real mutton, but their aromatic volatiles are possibly distinct in terms of category, quantity, and composition. Thus, volatile compound evaluation is prioritized in specific flavor studies, and the unique composition and content of various aromatic compounds in mutton can be used to identify mutton samples and serve as the standard in quality evaluation. Therefore, unlike conventional methods that focus mainly on determining certain whole compounds, flavor fingerprinting can characterize a complex system with quantitative reliability [7].

Chromatographic fingerprinting technique is a unique method used in distinguishing authentic from counterfeit products and in evaluating the quality of fruits, vegetables [8], tea [9], beverages [10], flavors, and fragrances [11], and 
so on. This technique was also used to evaluate the quality of meat products. For instance, Tian et al. [12] confirmed that the moderate oxidized suet may be a desirable precursor for mutton process flavors by using a PLSR model. Considered effective for accessing the quality of raw materials and/or their processing products, this technique is a system model that is established on the basis of data acquired using chromatographic techniques, thin-layer chromatography, Xray, gas chromatography (GC), high-performance liquid chromatography, and so on to establish the specific patterns of recognition for multiple compounds in the samples [13]. This technique is basically characterized by integrity and ambiguity [14]. Ambiguity emphasizes fingerprint similarity rather than identity between control and test samples, whereas integrity emphasizes the integral composition and features of sample components, that is, the integral chromatographic features. However, minor differences between the mutton and fraud meat products may not be observed because of the complexity of the analyzed samples. Nonetheless, pattern recognition methods, such as linear discriminant analysis (LDA), principal component analysis (PCA), partial least squares-discriminant analysis (PLS-DA), and softindependent modeling of class analogy, facilitate the visualization of the information in the fingerprints. In this research, a method was designed in which flavor fingerprints were used in PLS-DA and PCA to build a target pattern recognition system for the quality control of mutton.

This research was primarily aimed at determining the characteristic flavor fingerprint of mutton through GCMS and GC-O. Moreover, PCA and PLS-DA were used to distinguish mutton from other kinds of meat or adulterated products for the quality control of mutton. Considering the above-mentioned analysis, the new GC-MS/GC-O analysis described here coupled with chemometric methods is a promising methodology to ensure mutton quality and achieve comprehensive quality control for mutton.

\section{Materials and Methods}

2.1. Materials and Chemicals. Different batches of mutton samples obtained from different sources, as well as horsemeat samples, were provided by Beitun EHe steppe Food Co., Ltd. (Xinjiang, China). The sheep were reared indoors and were fed a maize silage-based diet. They had free access to hay and straw; they were slaughtered at $280 \mathrm{~d}$ of age. Pork, chicken, duck, and beef were purchased from the Wuxi Wal-Mart store (Wuxi, China). $\mathrm{C}_{6}-\mathrm{C}_{26}$, a mixture of serial $n$-alkanes, was obtained from Sinopharm Chemical Reagent Co., Ltd. (Shanghai, China). Reference components were purchased from Sigma Aldrich Co., Ltd. (Shanghai, China).

2.2. Sample Preparation. Bones were immediately removed from the mutton samples, which were divided into five parts for analysis (neck, forelegs, sternal rib, abdomen, and draw legs with buttocks). Each part weighed $500 \mathrm{~g}$ and was wrapped in aluminum foil. Parts were then stored at $-20^{\circ} \mathrm{C}$ for further analysis. Prior to analysis, the meat samples were defrosted for $24 \mathrm{~h}$ at $4^{\circ} \mathrm{C}$ and cut into $0.5 \mathrm{~cm} \times 0.5 \mathrm{~cm} \times$
$0.5 \mathrm{~cm}$ cubes. Each part of the meat samples was accurately weighed (100.0 g; total weight, $500.0 \mathrm{~g}$ ), mixed with $500 \mathrm{~mL}$ of tap water, and pressure-cooked (121 Pa) for $30 \mathrm{~min}$. The samples were then immediately cooled in ice water, sealed, and then stored at $-18^{\circ} \mathrm{C}$ for subsequent analysis.

2.3. GC-MS Analysis. The volatiles were sampled by using a $75 \mu \mathrm{m}$ CAR/PDMS SPME fiber (Supelco, USA). Three grams of the meat samples was distributed evenly in a $15 \mathrm{~mL}$ vial and then mixed by using a magnetic stirring bar. Prior to trapping, 1,2-dichlorobenzene $(4 \mu \mathrm{L}, 0.555 \mu \mathrm{g} / \mu \mathrm{L})$ used as an internal standard was added to each sample. The vial was closed and sealed by a PTFE/BYTL septum, placed in a $55^{\circ} \mathrm{C}$ water bath. Then the fiber was exposed in the sample headspace, maintained for $30 \mathrm{~min}$, and then transferred immediately into the GC injection port. Then the fiber was desorbed for $5 \mathrm{~min}$ at $250^{\circ} \mathrm{C}$.

Chromatographic separation was performed using a Finnigan trace GC-MS (Finnigan, USA) equipped with a FID detector. The volatile compounds were separated on a DB-WAX column (J \& W Scientific Inc., USA; $30 \mathrm{~m}$ length, $0.25 \mathrm{~mm}$ i.d., $0.25 \mu \mathrm{m} d_{f}$ ). The carrier gas was helium (99.999\%) and had a flow rate of $1.8 \mathrm{~mL} / \mathrm{min}$. The oven temperature was programmed to run for $3 \mathrm{~min}$ at $40^{\circ} \mathrm{C}$, subsequently increased to $80^{\circ} \mathrm{C}$ at $6^{\circ} \mathrm{C} / \mathrm{min}$, then increased to $230^{\circ} \mathrm{C}$ at $10^{\circ} \mathrm{C} / \mathrm{min}$, and finally held for $10 \mathrm{~min}$. The detection temperature was $250^{\circ} \mathrm{C}$. MS spectra were acquired under electron impact mode at an emission current of $35 \mathrm{~mA}$ and ionization voltage of $70 \mathrm{eV}$. The transfer line temperature was $250^{\circ} \mathrm{C}$, whereas the quadrupole mass filter was employed at $150^{\circ} \mathrm{C}$. A mass range of $\mathrm{m} / z=35$ to 450 was noted at the rate of $4.45 \mathrm{scan} / \mathrm{s}$.

Comparing the Kovats retention indices (KI) and the mass spectra of individual components with those of authentic standard compounds allowed identification of the volatile components. They were tentatively identified using WILEY and NIST and KI. The relative concentration of volatile components was calculated on the basis of their peak area with those of 1,2-dichlorobenzene. The expression of KI value is defined as follows:

$$
\mathrm{KI}(x)=100 n+\frac{\mathrm{RT}(x)-\mathrm{RT}(n)}{\mathrm{RT}(n+1)-\mathrm{RT}(n)} \times 100,
$$

where $x$ is the unknown compound and $n$ and $n+1$ are the number of carbon atoms of the $n$-alkanes eluted before and after the unknown compound $x$, respectively. $\operatorname{Rt}(x)$, $\mathrm{RT}(n)$, and $\mathrm{RT}(n+1)$ are the retention times of the unknown compound $x, n$-alkanes of carbon number $n$, and $n+1$, respectively.

2.4. GC-O Analysis. GC-O was conducted with Finnigan trace gas chromatograph equipped with an OP275 sniffing port (GL Sciences Inc., Japan) and an FID. The effluent from the DB-WAX column was divided at 1:1 ( $\mathrm{v} / \mathrm{v})$ between the sniffing port and FID. Nitrogen was the carrier gas and it had a flow velocity of $2.1 \mathrm{~mL} / \mathrm{min}$. The samples were collected, and the column and analyzing conditions were all similar to those in GC-MS. A detection frequency method involving 8 welltrained panelists (4 males and 4 females, aged range 26-48) 
was used to acquire the odor profile of mutton samples. The method was used as previously described [15].

2.5. Statistical Data Processing. ANOVA was carried out using SPSS18.0. PCA and PLS-DA were conducted using Unscrambler $\mathrm{X}$ version 9.6. The chemometric methods were performed using the average of 3 gas chromatographic peaks that were acquired from triplicates of each sample.

Repeatability, precision, and stability were evaluated by calculating their relative standard deviations (RSDs) to validate analytical method for the flavor fingerprinting. Repeatability was assessed by analyzing 5 different preparations of the same sample. Precision was evaluated by reduplicating continuous injections of the same sample for 5 times. Regarding sample stability, it was performed by analyzing the same treated sample that was stored for $36 \mathrm{~h}$ at RT.

The common volatile compounds in the fingerprints acquired through GC-MS were analyzed using PCA to obtain a visual representation of the similarity among samples. First, thirty-two characteristic compounds of mutton flavor fingerprint were extracted from the mutton samples. The data for the compound were recorded as 0 if this compound was not detected in one sample. Then, the dataset for the analytical samples was performed to be the input data through mean centering of the 32 common characteristic components in place of the full spectrum of the chromatogram. Then, this dataset was integrated into the map matrix for further analysis. In this part of the method, PCA was carried out to distinguish and identify different meat or adulterated samples. The samples included 25 mutton samples, 5 beef samples, five chicken samples, 5 pork samples, 5 horse meat samples, 5 duck samples, and 5 fraud mutton samples. Each sample was analyzed in triplicate under similar conditions. The sample data matrices were denoted as M1-25 (mutton), B1-5 (beef), C1-5 (chicken), P1-5 (pork), H1-5 (horse meat), D1-5 (duck), and F1-5 (fraud samples).

PLS-DA was conducted to predict the identity of the mutton samples by using the common volatile data. This technique combines PLSR and DA. Thus, this process demonstrates the advantages of PLS, that is, feature extraction and noise reduction, and is applicable particularly in situations involving few samples but numerous variables [16]. The samples analyzed and the methods for data processing in PLS-DA were similar to those in PCA. First, six of the above-mentioned known meat samples and fraud samples were selected for PLS modeling. Based on the fingerprint information, the classified variables of 25 random mutton samples, considered as training samples, were set as 1 , and those of the other samples were labeled as 0 . The samples in the training set were subsequently subjected to regression analysis to establish the PLS models of the chromatographic data and classified variables. The $X$-axis represents the 32 characteristic compounds, and the $Y$-axis represents the classified variables ( 1 or 0$)$. All variables were scaled and centered to $1 / \mathrm{Sedv}$. The PLS model was then validated using full cross-validation to set the unit variance and a zero mean for each variable before performing PLS analysis. This ensured an unbiased contribution of each variable to a criterion. The other 25 external mutton samples and 5 fraud mutton samples that were not used in constructing the model were predicted on the basis of the threshold value (ultimate result with \pm 0.5 deviation) calculated by the model. The mutton samples were identified according to a cut-off value of 0.5 . In other words, samples with prediction value of $>0.5$ and deviation of $<0.5$ were identified as mutton, and samples with prediction value of $<0.5$ and deviation of $<0.5$ were identified as other meats or fraud samples. When the deviation exceeded 0.5 , the established identification model was regarded as unstable.

\section{Results and Discussion}

3.1. Standardization of Chromatographic Fingerprint. A chromatographic fingerprint is a mutual pattern of several common compounds with some characteristics in all the analyzed samples. It should feature the fundamental attributes of fuzziness and integrity or differences and sameness. The mutton could be accurately authenticated and identified (integrity) with these fingerprints even if the concentrations of the characteristic chemical components were not exactly similar among samples (fuzziness). Thus, the most important aspect of building a reliable fingerprint and standardizing its information feature is the extraction and quantification of the common characteristic compounds. Twenty-five mutton samples (M1-M25) were analyzed by GC-MS using the same experimental conditions.

Generally, the average chromatograms are considered standardized characteristic fingerprint information, and this was also partly adopted in this study. In this study, 162 peaks were detected but only 73 existed in all mutton samples. These 73 coexistent compounds are considered the "common components" used to establish a fingerprint. For flavor fingerprints, however, the amount of a compound did not necessarily reflect the intensity of the perceived aroma due to the different odor thresholds for distinct compounds. Therefore, contrary to traditional fingerprints, the common characteristic compounds in flavor fingerprint should also be odor-active, which can be tested through GC$\mathrm{O}$ assessment with a detection frequency of $>50 \%$. Based on the detection frequency results, only 32 compounds possessed odor activities. Thus, these compounds can be assigned as the common characteristic compounds. Then, the methods used in quantifying the above 32 common compounds to establish a standard fingerprint require further investigation. However, several phenomena, especially for the changes in the response values of the peaks during data processing, may reduce the accuracy of the fingerprints. This problem can usually be resolved by selecting a stable peak as reference peak or adding an internal standard substance. In this study, 1,2-dichlorobenzene was chosen to be an internal standard when calculating the relative concentrations. The average chromatographic information of selected common odor-active components was regarded as the standardized characteristic flavor fingerprint of mutton, according to this method (Table 1). 
TABLE 1: The information of chromatographic fingerprint of active-odor compounds of mutton.

\begin{tabular}{|c|c|c|c|c|c|}
\hline Number $^{\mathrm{a}}$ & Compound & $\mathrm{KI}^{\mathrm{b}}$ & Relative concentration $^{c}$ & Odor description & $\mathrm{ID}^{\mathrm{d}}$ \\
\hline 1 & 3-Methylbutanal & 986 & $13.38 \pm 0.22$ & Nutty, chocolate, caramel & $\mathrm{A}$ \\
\hline 2 & Hexanal & 1092 & $101.92 \pm 6.11$ & Fruity, fatty, green & $\mathrm{A}$ \\
\hline 3 & Heptanal & 1209 & $43.32 \pm 2.01$ & Nutty, oily, fruity & A \\
\hline 4 & Octanal & 1307 & $24.35 \pm 0.82$ & Fruity, floral, fatty & $\mathrm{A}$ \\
\hline 5 & Nonanal & 1411 & $18.95 \pm 0.59$ & Oily, fatty & A \\
\hline 6 & 2-Octenal & 1429 & $3.59 \pm 0.71$ & Fruity & $\mathrm{C}$ \\
\hline 7 & Decanal & 1582 & $11.18 \pm 0.38$ & Rubber tubing, smoky & $\mathrm{A}$ \\
\hline 8 & (E)-2-nonenal & 1616 & $50.12 \pm 0.56$ & Tallowy, green & $\mathrm{B}$ \\
\hline 9 & Benzaldehyde & 1622 & $8.76 \pm 0.61$ & Nutty & $\mathrm{C}$ \\
\hline 10 & (E,E)-2,4-octadienal & 1776 & $10.02 \pm 0.26$ & Fatty, boiled meat & $\mathrm{C}$ \\
\hline 11 & (E)-2-decenal & 1812 & $11.80 \pm 0.91$ & Rosin, green & $\mathrm{B}$ \\
\hline 12 & 2-Undecenal & 1836 & $24.13 \pm 1.96$ & Green, fatty & A \\
\hline 13 & Tetradecanal & 1873 & $11.32 \pm 2.05$ & Buttery, fruity, green & A \\
\hline 14 & Octadecanal & 2314 & $3.89 \pm 0.35$ & Fatty, candle & A \\
\hline 15 & 1-Octen-3-ol & 1435 & $15.34 \pm 0.89$ & Mushroom & A \\
\hline 16 & Hexanol & 1426 & $10.28 \pm 1.63$ & Green, fruity, oily & $\mathrm{A}$ \\
\hline 17 & 3-Octanol & 1566 & $11.08 \pm 0.67$ & Candle, elecampane & A \\
\hline 18 & Acetic acid & 1543 & $8.11 \pm 0.37$ & Pungency, sour & A \\
\hline 19 & 4-Ethyloctanoic acid & 1998 & $153.29 \pm 16.82$ & Cheese, boiled mutton & $\mathrm{A}$ \\
\hline 20 & Hexoic acid & 2006 & $19.91 \pm 1.38$ & Lamb, oily & A \\
\hline 21 & 4-Methylnonanoic acid & 2261 & $296.52 \pm 16.14$ & Tallowy, roasted, mutton & A \\
\hline 22 & Nonanoic acid & 2309 & $32.18 \pm 1.27$ & Fatty, cheese & A \\
\hline 23 & Decanoic acid & 2407 & $12.06 \pm 0.53$ & Rancid, oily & A \\
\hline 24 & 6-Methyl-5-hepten-2-one & 1441 & $42.78 \pm 1.12$ & Meaty, tallowy & B \\
\hline 25 & 2-Undecanone & 1711 & $14.98 \pm 0.22$ & Oily, fruity & $\mathrm{B}$ \\
\hline 26 & 2-Pentyl decanone & 1772 & $36.33 \pm 2.31$ & Fatty & $\mathrm{B}$ \\
\hline 27 & 2-Pentadecanone & 2040 & $7.08 \pm 0.91$ & Oily & $\mathrm{C}$ \\
\hline 28 & Ethyl dodecanoate & 2053 & $9.31 \pm 0.42$ & Wax, fruity, fatty & A \\
\hline 29 & 2-Pentylfuran & 1280 & $29.19 \pm 0.82$ & Earthy, meaty & A \\
\hline 30 & 2-Methyl-5-(methylthio)furan & 1455 & $29.19 \pm 3.62$ & Roasted, peanut & A \\
\hline 31 & 4-Methyl-5-thiazoleethanol & 2443 & $18.38 \pm 0.52$ & Meaty, roasted, nutty & $\mathrm{C}$ \\
\hline 32 & 2,3,5,6-Tetramethyl pyrazine & 2207 & $19.37 \pm 0.51$ & Roasted, meaty & $\mathrm{A}$ \\
\hline
\end{tabular}

${ }^{a}$ Code representing the 32 active-odor compounds observed in GC-MS-O analysis; ${ }^{b}$ KI: Kovats index in column DB-Wax; ${ }^{c}$ values reported are means \pm S.D. $(\mu \mathrm{g} / \mathrm{kg})$; ${ }^{\mathrm{d}}$ identification method: (A) mass spectrum and KI agree with that of the authentic compound run under similar GC-MS conditions; (B) tentative identification based on interpretation of mass spectrum and comparison with similar compound. (C) Mass spectrum and KI agree with literature data or NIST Chemistry WebBook (http://webbook.nist.gov/chemistry/name-ser/).

Among the compounds mentioned in Table 1, three have been determined as the decisive characteristic flavor compounds of mutton: 4-ethyloctanoic acid (cheese, boiled mutton), 4-methylnonanoic acid (tallow, roasted, mutton), and hexanoic acid (lamb, oily) [6]. Moreover, some aliphatic aldehydes, especially some short-chain aliphatic aldehydes $\left(\mathrm{C}_{5}-\mathrm{C}_{9}\right)$, were also reported in sheep meat; these aldehydes are as follows: hexanal (fruity, fatty, and green), heptanal (nutty, oily, and fruity), octanal (fruity, floral, and fatty), nonanal (oily, fatty), and 2-octenal (fruity) [17]. Furthermore, 6-methyl-5-hepten-2-one, 3-methylbutanal, 2-methyl5-(methylthio) furan, 2-undecenal, and 2,3,5,6-tetramethyl pyrazine were related to emitting meaty odors, and all of these components influence the overall mutton flavor to a certain extent. 4-Methyloctanoic acid is described as the odor of mutton [18], but it was not detected in this study.

The method was then validated regarding its repeatability, precision, and stability. The instrument/injection precision of the method, as represented by RSD, was lower than $1.02 \%$ for relative retention time (RRT) and within $0.28 \%$ to $2.96 \%$ for relative peak area (RPA). The RSD of the repeatability of RRT and RPA were lower than $0.64 \%$ and $3.78 \%$, respectively. In addition, sample stability, which was also evaluated by RSD, ranged from $0.21 \%$ to $1.32 \%$ for RRT and from $0.79 \%$ to $5.01 \%$ 


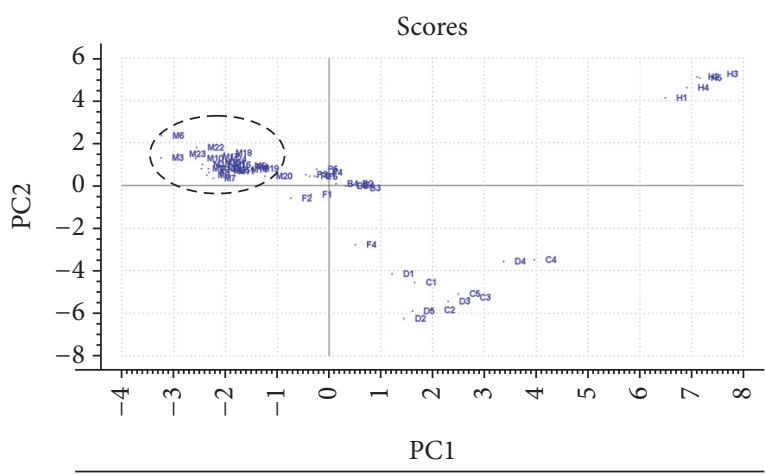

Result 1, $X$-expl: 26\%,24\%

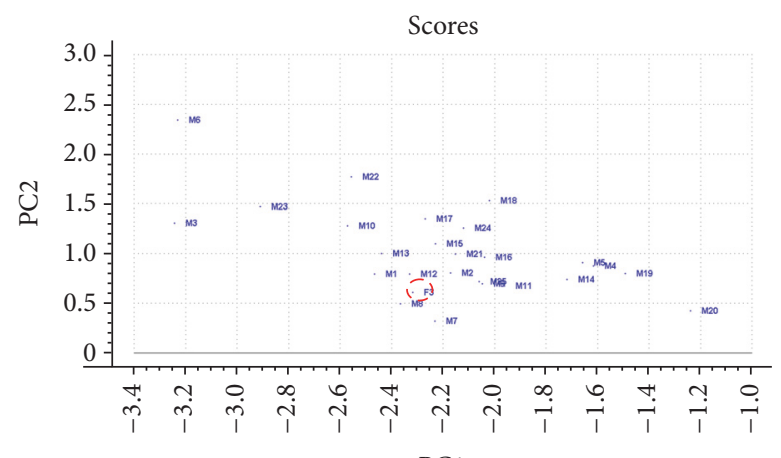

PC1

(a)

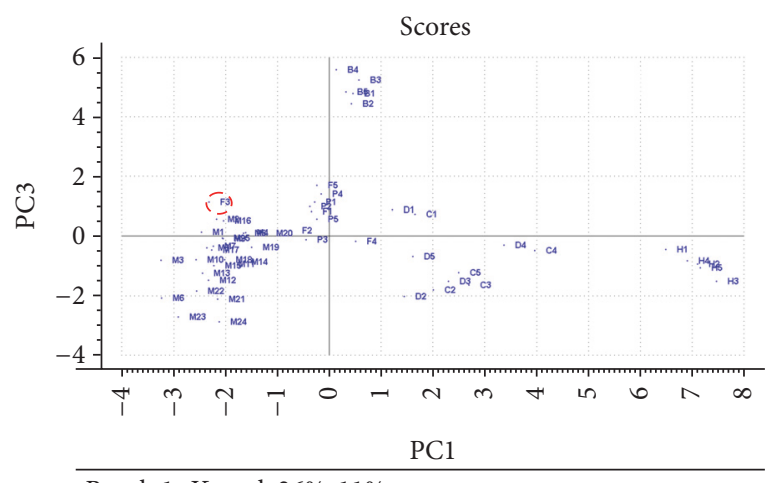

Result 1, $X$-expl: $26 \%, 11 \%$

(c)

FIGURE 1: Principal component projections for the 25 mutton samples (M1-M25), 5 fraud samples (F1: pork mixed with synthetic simulated mutton flavor; $\mathrm{F}_{2}$ : duck mixed with synthetic simulated mutton flavor; $\mathrm{F}_{3}$ : pork lean mixed with mutton fat; $\mathrm{F}_{4}$ : duck mixed with $20 \%$ lean mutton; $\mathrm{F}_{5}$ : pork mixed with $20 \%$ lean mutton), and 25 other kinds of meat samples (D1-D5: duck samples; C1-C5: Chicken samples; H1-H5: horsemeat samples; B1-B5: beef samples; P1-P5: pork samples). (a) PC1-PC2; (b) enlargement of the dashed line and spot line in figure (a); (c) PC1-PC3.

for RPA. These results showed that the method is applicable and reliable for the analysis and establishment of the mutton flavor fingerprint and subsequent analysis.

\subsection{Application of the Fingerprint in Quality Control of} Mutton. The developed mutton flavor fingerprint contained information that discriminates mutton samples from other meat samples and from adulterated samples as verified by the PCA and PLS-DA loadings.

3.3. PCA. The derived PCA showed three significant PCs, which explained $61 \%$ of the total variance. Thus, PC1-PC2 and PC1-PC3 were mapped (Figure 1). According to the fundamentals of PCA, proximity to score plots is associated with high similarity with respect to the two series of components concerned; otherwise, they differ from each other [19]. As shown in Figure 1, it is observed that 25 mutton samples and 25 other meat samples were located in different positions, which indicated that the samples significantly differed in two-dimensional space projection. For adulterated mutton samples, $\mathrm{F}_{1}$ (pork mixed with artificial mutton flavor), $\mathrm{F}_{2}$ (duck mixed with synthetic simulated mutton flavor), $\mathrm{F}_{4}$ (duck mixed with $20 \%$ lean mutton), and $\mathrm{F}_{5}$ (pork mixed with $20 \%$ lean mutton) were easily distinguished from mutton samples. However, $\mathrm{F}_{3}$ (lean pork mixed with mutton suet) are nearly similar to mutton samples (Figure 1(b)). This result demonstrated that sample pork mixed with mutton fat can produce a flavor similar to that of normal mutton. This phenomenon may be attributed to the fact that the characteristic flavor of mutton is mainly attributed to mutton suet, and lean meat only provides the basic meat flavor. This result is similar to those of other studies. Wasserman and Talley (1968) found that when beef tallow was added to veal, evaluators could distinguish veal from beef; however, when lamb fat was added to veal, veal is more easily mistaken as lamb. The above results indicated that PCA performed based on fingerprint information is generally useful in the quality control of mutton, but it is limited for adulterated samples, particularly for samples mixed with mutton suet.

3.4. PLS-DA. Considering the above results, an alternative strategy can be performed, which is using a supervised classification technique, such as PLS-DA. The data matrix in the PLS-DA model was comprised of 55 rows (samples in the 


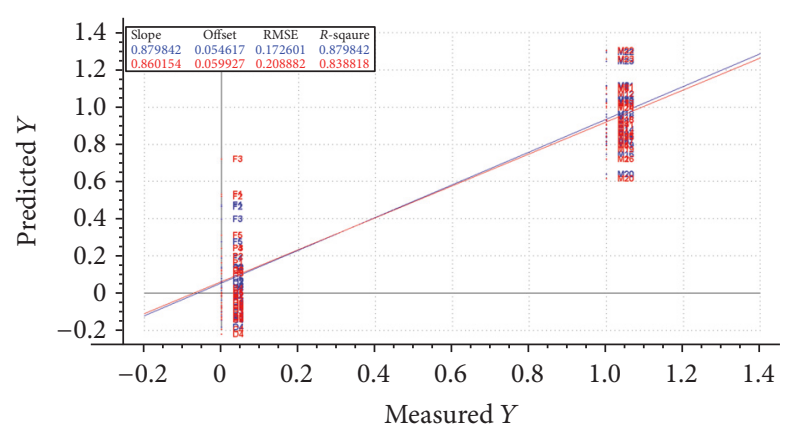

Result 2, ( $Y$-var, PC): (M/other, 2) (M/other, 2)

FIGURE 2: Predicted versus measured values among mutton samples, fraud samples, and other meats from PLS-DA model. The labels of the samples correspond to the code in Figure 1.

training set) and 32 columns (data on common characteristic volatiles). Figure 2 showed the plot correlating predicted versus measured classified variables is a reasonably good predictor of mutton samples in most cases. The root-meansquare error for the prediction was 0.209 (lower than the error standard $<0.5)$, and the explained variance was $0.880(83.9 \%$ by cross-validation). These results revealed that the PLSDA model achieved a good fitting without overfitting and is thus applicable in mutton sample classification and identification.

The observations in the different groups of the prediction set, namely, mutton, other meats, and fraud mutton samples, were accurately clustered close to their corresponding groups in the PLS-DA model (Figure 3(a)). The model achieved was validated through an external test set of samples to test the stability of PLS-DA. The identification and prediction results are shown in Figure 4, whereas the specific analytical data are shown in Table 2 . The predicted $Y$ scores of the 25 external mutton samples achieved prediction values of $>0.640$ (latent variable 1 ) with deviation of $<0.22$, whereas the adulterated samples, which were analyzed via a method similar to PCA, showed prediction values of $0.189-0.473$ with deviation of $<0.31$. According to the PLS-DA fundamentals, mutton samples can be identified correctly by using the PLS-DA model, indicating that the proposed model can sufficiently discriminate mutton and other samples.

Furthermore, PLS-DA loadings were graphed to identify the possible volatiles that contributed to sample discrimination. Loadings (E)-2-nonenal (tallowy, green), (E)-2decenal (rosin, green), (E,E)-2,4-octadienal (fatty, boiled meat), tetradecanal (buttery, fruity, green), octadecanal (fatty, candle), acetic acid (pungency, sour), 4-ethyloctanoic acid (cheese, boiled mutton), decanoic acid (rancid, oily), nonanoic acid (fatty, cheese), 2-undecanone (oily, fruity), 2-pentadecanone (oily), ethyl dodecanoate (wax, fruity, fatty), 2-pentylfuran (earthy, meaty), and 2-methyl-5-(methylthio)furan (roasted, peanut) were the variables that contributed the most to sample discrimination (Figure 3(b)). These 14 compounds should be used as chemical markers for discriminating the internal quality mutton samples from that of the other meat samples, which would indicate that the

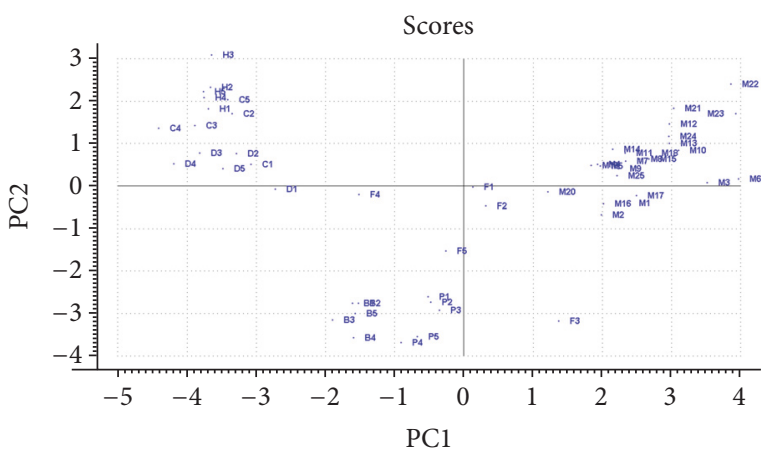

Result 2, X-expl: 24\%, 11\% Y-expl: 78\%, 10\%

(a)

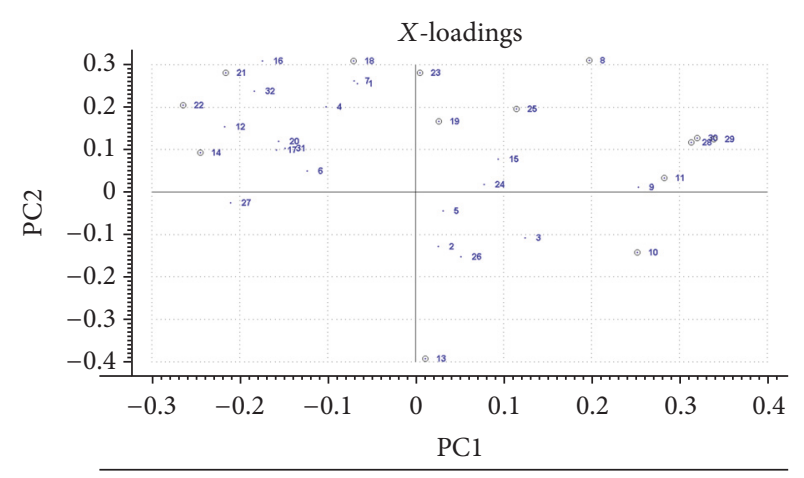

Result 1, X-expl: 24\%, 11\% Y-expl: 78\%, 10\%

(b)

Figure 3: PLS-DA scores (a) and loadings (b) plots among mutton samples, fraud samples, and other meats. The labels of the samples correspond to the code in Figure 1. The labels of the loadings correspond to the code in Table 1.

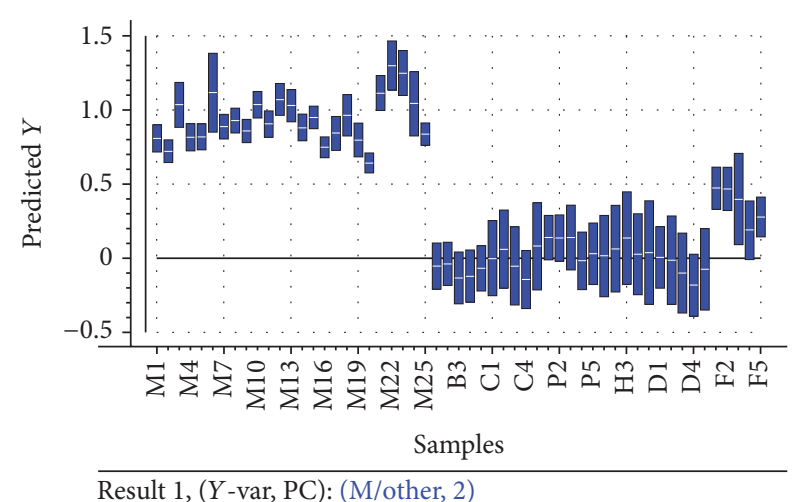

FIGURE 4: Discriminant results for validation set of samples by PLSDA model. M1-M25, 25 mutton samples that did not participate in the modeling. The labels of 5 fraud samples and 25 other kinds of meat samples correspond to the code in Figure 3, but do not participate in the modeling.

quality of mutton samples could be assessed quantitatively by determining the amounts of these chemical markers. Thus, the above-mentioned compounds possibly influence the discrimination of mutton samples. 
TABLE 2: Discriminant data for calibration set of mutton samples, fraud mutton samples, and other meat samples by PLS-DA model.

\begin{tabular}{|c|c|c|}
\hline Sample* & Prediction & Deviation \\
\hline M1 & 0.806 & 0.091 \\
\hline M2 & 0.719 & 0.077 \\
\hline M3 & 1.034 & 0.152 \\
\hline M4 & 0.814 & 0.090 \\
\hline M5 & 0.817 & 0.088 \\
\hline M6 & 1.116 & 0.266 \\
\hline M7 & 0.887 & 0.083 \\
\hline M8 & 0.926 & 0.083 \\
\hline M9 & 0.856 & 0.078 \\
\hline M10 & 1.035 & 0.089 \\
\hline M11 & 0.904 & 0.089 \\
\hline M12 & 1.069 & 0.107 \\
\hline M13 & 1.028 & 0.108 \\
\hline M14 & 0.881 & 0.090 \\
\hline M15 & 0.947 & 0.077 \\
\hline M16 & 0.747 & 0.070 \\
\hline M17 & 0.841 & 0.115 \\
\hline M18 & 0.962 & 0.138 \\
\hline M19 & 0.797 & 0.113 \\
\hline M20 & 0.640 & 0.067 \\
\hline M21 & 1.113 & 0.119 \\
\hline M22 & 1.297 & 0.165 \\
\hline M23 & 1.247 & 0.150 \\
\hline $\mathrm{M} 24$ & 1.042 & 0.215 \\
\hline M25 & 0.837 & 0.077 \\
\hline B1 & -0.053 & 0.156 \\
\hline B2 & -0.039 & 0.145 \\
\hline B3 & -0.135 & 0.174 \\
\hline B4 & -0.122 & 0.177 \\
\hline B5 & -0.069 & 0.152 \\
\hline $\mathrm{C} 1$ & -0.003 & 0.252 \\
\hline $\mathrm{C} 2$ & 0.059 & 0.264 \\
\hline $\mathrm{C} 3$ & -0.053 & 0.261 \\
\hline $\mathrm{C} 4$ & -0.145 & 0.196 \\
\hline $\mathrm{C} 5$ & 0.078 & 0.293 \\
\hline $\mathrm{P} 1$ & 0.138 & 0.148 \\
\hline $\mathrm{P} 2$ & 0.134 & 0.157 \\
\hline P3 & 0.138 & 0.218 \\
\hline $\mathrm{P} 4$ & -0.020 & 0.194 \\
\hline P5 & 0.030 & 0.207 \\
\hline H1 & 0.013 & 0.274 \\
\hline $\mathrm{H} 2$ & 0.063 & 0.293 \\
\hline H3 & 0.134 & 0.298 \\
\hline $\mathrm{H} 4$ & 0.027 & 0.273 \\
\hline H5 & 0.037 & 0.350 \\
\hline D1 & 0.003 & 0.208 \\
\hline D2 & -0.015 & 0.298 \\
\hline D3 & -0.101 & 0.269 \\
\hline
\end{tabular}

TABLE 2: Continued.

\begin{tabular}{lcc}
\hline Sample $^{*}$ & Prediction & Deviation \\
\hline D4 & -0.183 & 0.209 \\
D5 & -0.077 & 0.276 \\
F1 & 0.473 & 0.142 \\
F2 & 0.464 & 0.145 \\
F3 & 0.396 & 0.307 \\
F4 & 0.189 & 0.196 \\
F5 & 0.276 & 0.133 \\
\hline
\end{tabular}

${ }^{*}$ The labels of the samples correspond to the code in Figure 4.

\section{Conclusions}

GC-MS combined with GC-O was established for flavor fingerprint analysis to monitor the quality of mutton. Information on the common flavor fingerprint was acquired from 32 aroma-active volatile compounds present in 25 mutton samples. The results were then validated for the sample stability, repeatability, and reproducibility. The developed flavor fingerprint is appropriate for differentiating and identifying mutton samples by geographical origin when used in combination with the appropriate chemometric method (PLS-DA). This method can thus be used for quality control.

\section{Disclosure}

First author is Ping Zhan.

\section{Conflicts of Interest}

The authors have declared no conflicts of interest.

\section{Acknowledgments}

This research was conducted with support from National Key Research and Development Program of China (Grant no. 2016YFD0400705), National Natural Science Foundation of China (Grant no. 31460408), and China Postdoctoral Science Foundation Funded Project (Grant no. 2016M591029). The authors would like to thank Professor Liu Jingsheng for their critical help in the design of research content.

\section{References}

[1] V. Banskalieva, T. Sahlu, and A. L. Goetsch, "Fatty acid composition of goat muscles and fat depots: a review," Small Ruminant Research, vol. 37, no. 3, pp. 255-268, 2000.

[2] Y.-C. Huang, H.-J. Li, Z.-F. He, T. Wang, and G. Qin, "Study on the flavor contribution of phospholipids and triglycerides to pork," Food Science and Biotechnology, vol. 19, no. 5, pp. 1267$1276,2010$.

[3] J. D. Wood, M. Enser, A. V. Fisher et al., "Fat deposition, fatty acid composition and meat quality: a review," Meat Science, vol. 78, no. 4, pp. 343-358, 2008.

[4] F. B. Whitfield and D. S. Mottram, "Volatiles from interactions of maillard reactions and lipids," Critical Reviews in Food Science and Nutrition, vol. 31, no. 1-2, pp. 1-58, 1992. 
[5] M. M. Sutherland and J. M. Ames, "The effect of castration on the headspace aroma components of cooked lamb," Journal of the Science of Food and Agriculture, vol. 69, no. 4, pp. 403-413, 1995.

[6] V. C. Resconi, M. M. Campo, F. Montossi, V. Ferreira, C. Sañudo, and A. Escudero, "Relationship between odour-active compounds and flavour perception in meat from lambs fed different diets," Meat Science, vol. 85, no. 4, pp. 700-706, 2010.

[7] S. Song, X. Zhang, K. Hayat, Z. Xiao, Y. Niu, and K. Eric, "Coordinating fingerprint determination of solid-phase microextraction/gas chromatography-mass spectrometry and chemometric methods for quality control of oxidized tallow," Journal of Chromatography A, vol. 1278, pp. 145-152, 2013.

[8] A. Z. Berna, S. Buysens, C. Di Natale, I. U. Grün, J. Lammertyn, and B. M. Nicolaï, "Relating sensory analysis with electronic nose and headspace fingerprint MS for tomato aroma profiling," Postharvest Biology and Technology, vol. 36, no. 2, pp. 143-155, 2005.

[9] M. Dumarey, I. Smets, and Y. Vander Heyden, "Prediction and interpretation of the antioxidant capacity of green tea from dissimilar chromatographic fingerprints," Journal of Chromatography B: Analytical Technologies in the Biomedical and Life Sciences, vol. 878, no. 28, pp. 2733-2740, 2010.

[10] B. J. Savary, P. Vasu, A. Nunez, and R. G. Cameron, "Identification of thermolabile pectin methylesterases from sweet orange fruit by peptide mass fingerprinting," Journal of Agricultural and Food Chemistry, vol. 58, no. 23, pp. 12462-12468, 2010.

[11] X. Zhu, Y. Gao, Z. Chen, and Q. Su, "Development of a chromatographic fingerprint of tobacco flavor by use of GC and GC-MS," Chromatographia, vol. 69, no. 7-8, pp. 735-742, 2009.

[12] H. Tian, P. Zhan, W. Li et al., "Contribution to the aroma characteristics of mutton process flavor from oxidized suet evaluated by descriptive sensory analysis, gas chromatography, and electronic nose through partial least squares regression," European Journal of Lipid Science and Technology, vol. 116, no. 11, pp. 1522-1533, 2014.

[13] T. Cajka, K. Riddellova, M. Tomaniova, and J. Hajslova, "Recognition of beer brand based on multivariate analysis of volatile fingerprint," Journal of Chromatography A, vol. 1217, no. 25, pp. 4195-4203, 2010.

[14] B. Bertrand, R. Boulanger, S. Dussert et al., "Climatic factors directly impact the volatile organic compound fingerprint in green Arabica coffee bean as well as coffee beverage quality," Food Chemistry, vol. 135, no. 4, pp. 2575-2583, 2012.

[15] P. Zhan, H. Tian, X. Zhang, and L. Wang, "Contribution to aroma characteristics of mutton process flavor from the enzymatic hydrolysate of sheep bone protein assessed by descriptive sensory analysis and gas chromatography olfactometry," Journal of Chromatography B: Analytical Technologies in the Biomedical and Life Sciences, vol. 921-922, pp. 1-8, 2013.

[16] S. Mildner-Szkudlarz, H. H. Jeleń, and R. Zawirska-Wojtasiak, "The use of electronic and human nose for monitoring rapeseed oil autoxidation," European Journal of Lipid Science and Technology, vol. 110, no. 1, pp. 61-72, 2008.

[17] F. Caporaso, J. D. Sink, P. S. Dimick, C. J. Mussinan, and A. Sanderson, "Volatile flavor constituents of ovine adipose tissue," Journal of Agricultural and Food Chemistry, vol. 25, no. 6, pp. 1230-1234, 1977.

[18] E. Wong, C. B. Johnson, and L. N. Nixon, "The contribution of 4-methyloctanoic (hircinoic) acid to mutton and goat meat flavour," New Zealand Journal of Agricultural Research, vol. 18, no. 3, pp. 261-266, 1975.
[19] H. Tian, P. Zhan, Z. Deng, H. Yan, and X. Zhu, "Development of a flavour fingerprint by GC-MS and GC-O combined with chemometric methods for the quality control of Korla pear (Pyrus serotina Reld)," International Journal of Food Science and Technology, vol. 49, no. 12, pp. 2546-2552, 2014. 

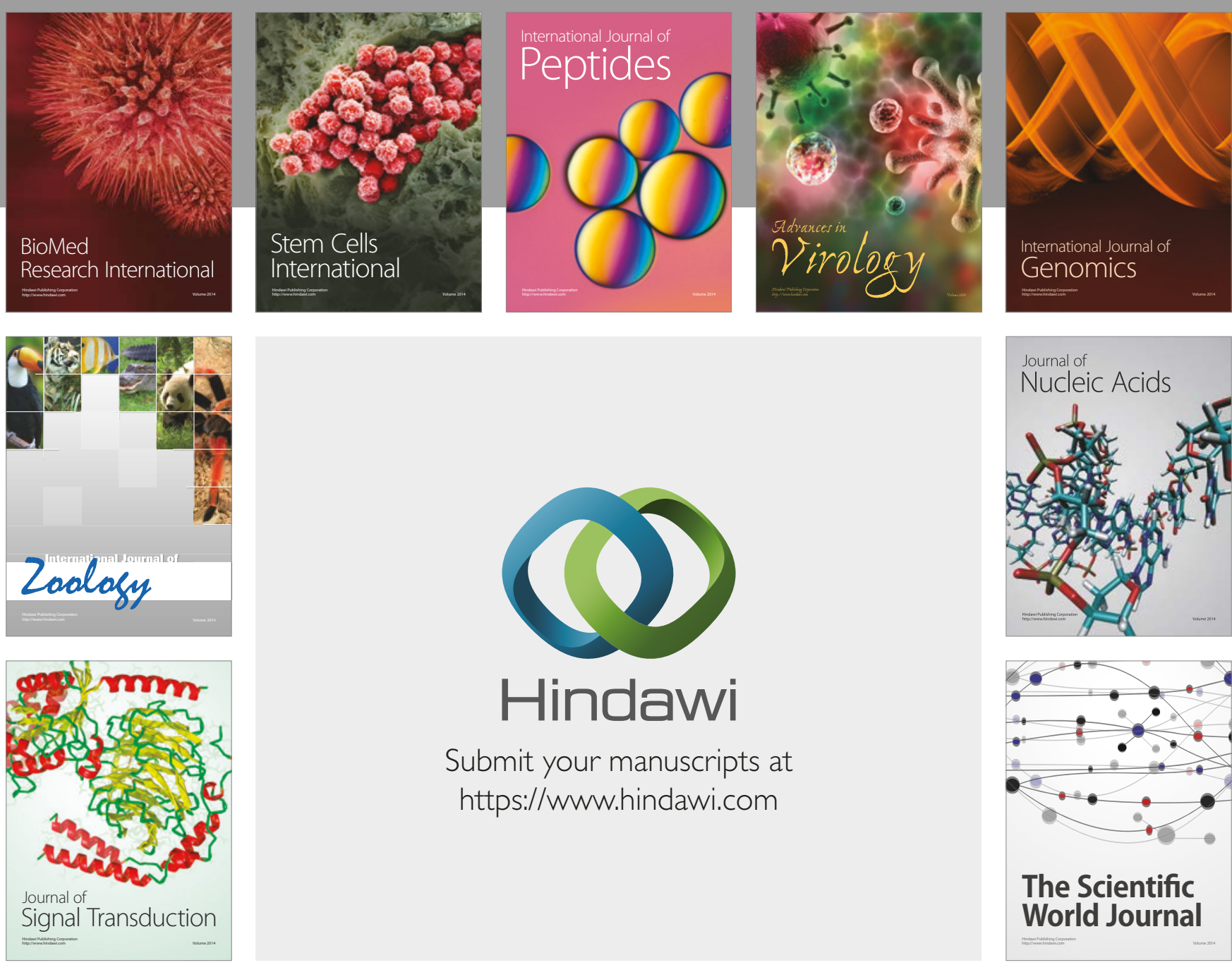

Submit your manuscripts at

https://www.hindawi.com
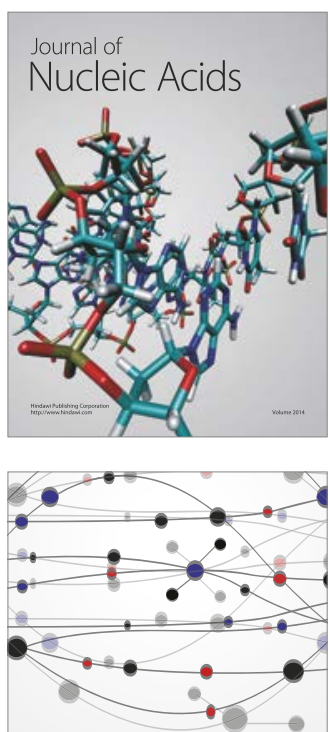

The Scientific World Journal

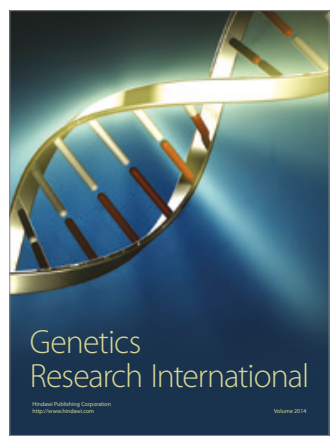

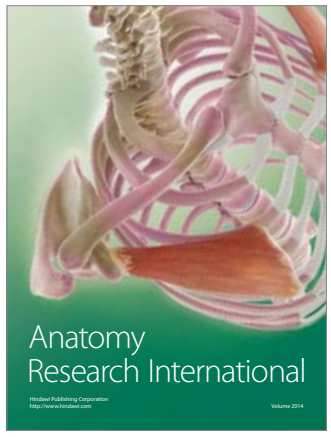

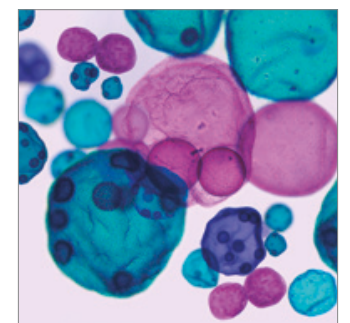

International Journal of Microbiology
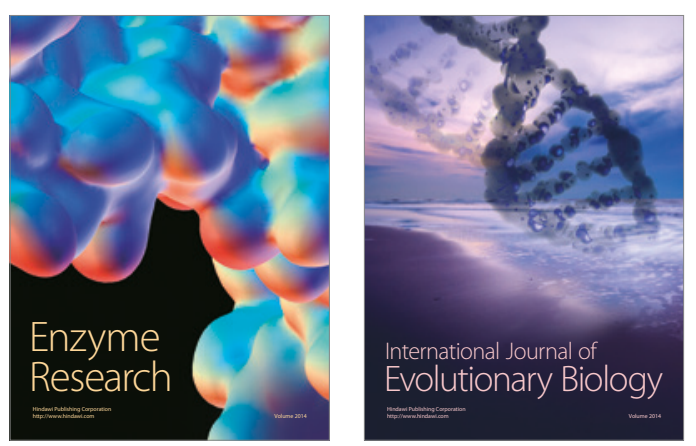
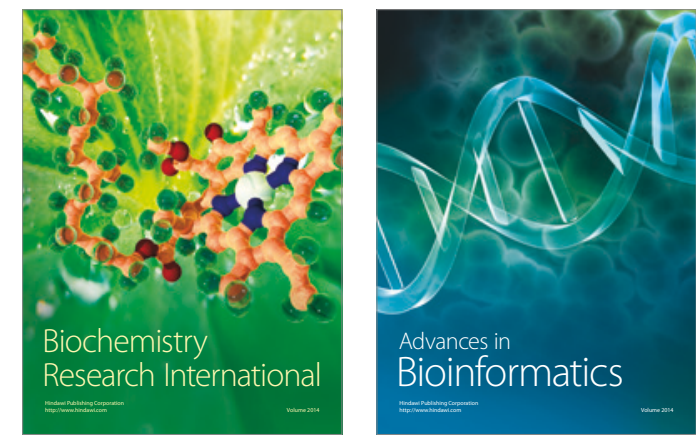

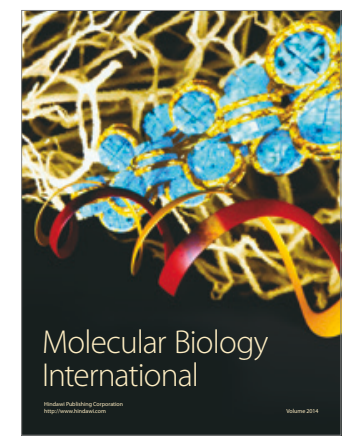

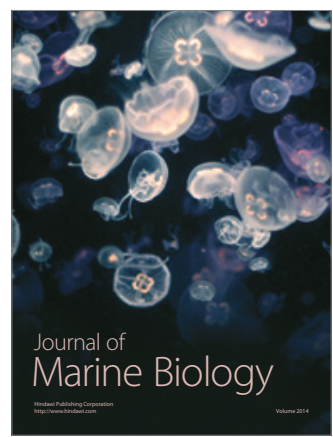

